\title{
Low biochemical nutritional parameters in acutely ill hospitalized elderly patients with and without stage III to IV pressure ulcers
}

\author{
Michael Gengenbacher, Hannes B. Stähelin, André Scholer, and Walter O. Seiler \\ Geriatric University Clinic and Clinical Chemical Laboratory, Kantonsspital, Basel, Switzerland
}

ABSTRACT. Background and aims: Pressure ulcers are associated with impaired nutritional status in acutely ill elderly patients. The objective of this study was to establish whether a difference exists between biochemical nutritional parameters in acutely ill elderly with stage III to IV pressure ulcers and a group of acutely ill elderly with no pressure ulcers. Methods: In a retrospective study we compared 8 biochemical nutritional markers in a group of 22 acutely ill elderly patients consecutively admitted to the geriatric ward who had stage III to IV pressure ulcers (PU group) in addition to their acute illness with a control group of 40 acutely ill elderly patients with no pressure ulcers (NPU group). Results: The PU group compared with the NPU group had significantly lower $(\mathrm{p}<0.0001)$ values of albumin, transferrin, hemoglobin, cholesterol, iron, and zinc $(\mathrm{p}<0.0059)$. Total lymphocyte count was slightly, but not significantly lower in the $P U$ group. In contrast, C-Reactive Protein levels were significantly higher $(\mathrm{p}<0.0001)$ in the $P U$ group compared with the NPU group, indicating a more severe illness in the presence of additional pressure ulcers. Conclusions: In this study, serum levels of biochemical nutritional parameters in acutely ill elderly patients with stage III to IV pressure ulcers are lower than those of acutely ill elderly subjects with no pressure ulcers, indicating a worse nutritional status of the PU patients. These findings, while not documenting a causal relationship, suggest the need for routine nutritional assessment and support in older patients, especially those with pressure ulcers.

(Aging Clin Exp Res 2002; 14: 420-423)

${ }^{\circ}$ 2002, Editrice Kurtis

\section{INTRODUCTION}

Malnutrition is increasingly appreciated as the most frequent comorbid condition in ill elderly patients (1). Specific pressure ulcer risk factors studied in the ill elderly include low biochemical markers of nutritional status such as low serum albumin, serum cholesterol, and hemoglobin levels (2). Wound healing is complicated by the high prevalence of malnutrition, immobility, and systemic diseases (3). Pressure ulcers are associated with impaired nutritional status in acutely ill elderly patients. Since malnutrition is potentially reversible, providing adequate nutrition to patients with pressure ulcers is a promising treatment approach $(4,5)$.

The objective of this study was to establish whether a difference exists between biochemical nutritional parameters in acutely ill elderly patients with stage III to IV pressure ulcers and a group of acutely ill elderly subjects with no pressure ulcers.

\section{METHODS}

\section{Study population}

The collection of 12 biochemical nutritional markers on Day 1 after admission of patients to the ward has been routine for ten years at the Geriatric University Clinic in Basel. This retrospective study examined 8 biochemical nutritional parameters in 22 acutely ill elderly patients with stage III to IV pressure ulcers (PU) who had been admitted consecutively to the geriatric ward. Ulcer classification as proposed by Shea (6) was used. Biochemical nutritional parameters were also analyzed in a control group of 40 acutely ill elderly patients with no pressure ulcers (NPU), admitted consecutively at the same time period and with comparable comorbidity. The biographical and clinical characteristics of the study and control subjects are shown in Tables 1 and 2.

Key words: Albumin, elderly, iron, malnutrition, pressure ulcer, zinc.

Correspondence: W.O. Seiler, M.D., Geriatric University Clinic, Kantonsspital, CH-4031 Basel, Switzerland.

E-mail: Walter-O.Seiler@unibas.ch

Received May 23, 2001; accepted in revised form April 30, 2002. 
Table 1 - Biographical data of the study subjects (PU) and the controls (NPU).

\begin{tabular}{lcc}
\hline & PU & NPU \\
\hline Total no. (N) of subjects & $22(100 \%)$ & $40(100 \%)$ \\
$\quad$ Male & $6(27 \%)$ & $11(27 \%)$ \\
Female & $16(73 \%)$ & $29(73 \%)$ \\
Age (years), mean (male+female) & $80.3 \pm 8.1$ & $82.2 \pm 6.6$ \\
$\quad$ Male, mean \pm SD & $72.8 \pm 7.6$ & $78.7 \pm 5.1$ \\
Range & $53-82$ & $65-97$ \\
Female, mean $\pm S D$ & $83.1 \pm 6.9$ & $83.5 \pm 6.0$ \\
Range & $53-94$ & $68-92$ \\
\hline
\end{tabular}

PU: Pressure Ulcer Patients; acutely ill elderly patients with stage III to IV pressure ulcers. NPU: Non-Pressure Ulcer Patients (controls); acutely ill elderly patients without pressure ulcers but with comorbidity comparable to the PU subjects (Table 2).

\section{Biochemical nutritional parameters}

Eight biochemical laboratory tests that may reflect protein energy malnutrition were performed with certified methods using the following analyzers: Hitachi 917 and Baxter Advia 120.

Fasting venous blood samples of PU and NPU subjects were collected using routine procedures on the morning after overnight fasting on Day 1 after hospital admission. Blood specimens were collected in lithium heparinate tubes for the analysis of CRP, albumin, iron, cholesterol and transferrin. Blood specimens for the zinc analysis by atomic absorption spectroscopy Varian (7) were collected using zinc-free needles and metal-free lithium heparinate tubes. Blood specimens for the measurements of total lymphocyte count and hemoglobin concentration were collected in EDTA tubes. All blood specimens were brought to the laboratory within an hour.

\section{Statistical methods}

Data are expressed as mean $\pm \mathrm{SD}$ and ranges unless otherwise specified. Statistical differences among groups were tested using the unpaired $t$-test and the Wilcoxon test for CRP. P-values less than 0.05 were considered statistically significant. Stat View Version 4.0 (Abacus Concepts, Inc.) was used for statistical calculations.

\section{RESULTS}

\section{Study population}

Table 1 shows the biographical data of the study and control subjects. There was no significant age or gender difference between the PU and NPU groups. Clinical characteristics, as shown in Table 2 , were quite similar for both the study (PU) and the control (NPU) subjects, except for bed fast immobility in 41 and $0 \%$, for diabetes mellitus in 36 and 15\%, and for hyperthyroidism in 18 and $0 \%$, respectively, which were more frequent in the PU group. In contrast, mean age was higher, and coro- nary heart disease and carcinoma more frequent in the NPU group.

\section{Biochemical nutritional parameters}

Table 3 summarizes the results of the 8 biochemical plasma parameters.

C-Reactive Protein (CRP) was significantly higher $(p<0.0001)$ in the PU group $(79.8 \pm 65.8 \mathrm{mg} / \mathrm{L}$; range 6224) compared with that of the NPU group $(13.5 \pm 14.9$ $\mathrm{mg} / \mathrm{L}$; range 2-139). Plasma levels of albumin, transferrin, hemoglobin, total cholesterol and iron were significantly lower $(p<0.0001)$ in the PU group.

When malnutrition is classified as mild (albumin 34-30 $\mathrm{g} / \mathrm{L}$ ), moderate (albumin 29-26 g/L), or severe (albu$\mathrm{min}<26 \mathrm{~g} / \mathrm{L}$ ), only 5 of $22 \mathrm{PU}$ patients were in the normal range, 5 in the mild, 6 in the moderate and 6 in the severe range. In contrast, 29 of $40 \mathrm{NPU}$ patients were in the normal range, 9 in the mild, 1 in the moderate, and 1 in the severe group. Plasma zinc levels were significantly lower $(p<0.0059)$ in the PU group, and total lymphocyte count was slightly but not significantly lower in the PU group.

\section{DISCUSSION}

Acutely ill elderly patients suffer from malnutrition, and pressure ulcers have been associated with impaired nutritional status in these patients, although a causal relationship has not been documented. The aim of the present study was to compare 8 biochemical nutritional parameters of a group of acutely ill patients who had stage

Table 2 - Clinical characteristics of the PU and NPU subjects.

\begin{tabular}{lcc}
\hline & PU & NPU \\
& N (\%) & N (\%) \\
\hline Total no. of subjects & $22(100)$ & $40(100)$ \\
Pressure ulcer & $22(100)$ & $0(0)$ \\
Immobility, bed fast & $9(41)$ & $0(0)$ \\
Paresis / plegic states & $3(14)$ & $7(18)$ \\
Arthrosis & $5(23)$ & $8(20)$ \\
Parkinsonism & $4(18)$ & $4(10)$ \\
Diabetes mellitus & $8(36)$ & $6(15)$ \\
Hyperthyroidism & $4(18)$ & $0(0)$ \\
Coronary heart disease & $10(45)$ & $23(58)$ \\
Hypertension & $10(45)$ & $20(50)$ \\
Peripheral vascular disease & $6(27)$ & $7(18)$ \\
Venous disease & $2(9)$ & $2(5)$ \\
Renal insufficiency & $2(9)$ & $4(10)$ \\
COPD (pulmonary disease) & $3(14)$ & $8(20)$ \\
Peptic ulcer & $3(14)$ & $7(18)$ \\
Minor depression & $3(14)$ & $6(15)$ \\
Carcinoma & $1(5)$ & $5(13)$ \\
\hline
\end{tabular}

PU: Pressure Ulcer Patients; NPU: Non-Pressure Ulcer Patients (controls). 
Table 3 - Biochemical nutritional parameters of the PU and NPU subjects.

\begin{tabular}{|c|c|c|c|c|c|c|}
\hline & \multirow[b]{2}{*}{ Normal values } & \multicolumn{2}{|c|}{$\mathbf{P U}$} & \multicolumn{2}{|c|}{ NPU } & \multirow[b]{2}{*}{$p^{*}$} \\
\hline & & Mean \pm SD & Range & Mean \pm SD & Range & \\
\hline CRP & $<10 \mathrm{mg} / \mathrm{L}$ & $79.8 \pm 65.8$ & $6-224$ & $13.5 \pm 14.9$ & $2-139$ & $<0.0001$ \\
\hline Albumin & $35-52 \mathrm{~g} / \mathrm{L}$ & $28.8 \pm 6.1$ & $18-39$ & $36.1 \pm 3.9$ & $24-43$ & $<0.0001$ \\
\hline Transferrin & $2.3-4.5 \mathrm{~g} / \mathrm{L}$ & $1.8 \pm 0.5$ & $0.9-3.2$ & $2.4 \pm 0.5$ & $1.4-3.5$ & $<0.0002$ \\
\hline Cholesterol & $3.0-5.2 \mathrm{mmol} / \mathrm{L}$ & $4.0 \pm 1.0$ & $2.7-6.6$ & $5.3 \pm 1.1$ & $2.8-7.5$ & $<0.0001$ \\
\hline Iron & $9.5-33.8 \mu \mathrm{mol} / \mathrm{L}$ & $6.3 \pm 4.9$ & $1.9-21.2$ & $12.6 \pm 5.3$ & $4.3-24.6$ & $<0.0001$ \\
\hline Zinc & $10.7-22.9 \mu \mathrm{mol} / \mathrm{L}$ & $9.3 \pm 1.9$ & $6.3-11.9$ & $10.7 \pm 1.6$ & 8.9-14.8 & $<0.0059$ \\
\hline Hemoglobin & $12-16 \mathrm{~g} / \%$ & $11.1 \pm 1.7$ & $8.6-14.0$ & $13 \pm 1.7$ & $9.4-16.8$ & $<0.0002$ \\
\hline Lymphocyte $^{\mathrm{a}}$ & $>1500 / \mu \mathrm{L}$ & $1299 \pm 724$ & $575-3642$ & $1583 \pm 744$ & $452-4000$ & NS \\
\hline
\end{tabular}

PU: Pressure Ulcer Patients; NPU: Non-Pressure Ulcer Patients; CRP: C-Reactive Protein.

a) Total lymphocyte count per $\mu \mathrm{L} . \quad p^{*}: p$-value less than 0.05 was considered statistically significant; NS: non significant.

III to IV pressure ulcers in addition to their acute illness, with the nutritional parameters of a control group of acutely ill patients with no pressure ulcers.

C-Reactive Protein (CPR) levels were significantly higher in the PU group, which may be an effect of the ulcer or reflect a more serious underlying condition (8). Matsuyama (8) also found plasma CRP and fibrinogen significantly increased in PU patients suggesting an acute inflammatory reaction releasing proinflammatory cytokines.

CRP levels correlated positively with the severity of disease in this study and may indicate higher concentrations of catabolic cytokines, including interleukin-1 (IL-1), interleukin-6 (IL-6) and tumour necrosis factor alpha (TNF alpha), which are known to aggravate malnutrition and hypercatabolism in pressure ulcer patients $(9,10)$.

Zinc levels were significantly lower $(p<0.0059)$ in the PU group. Low zinc and prealbumin levels have been found in patients with PUs (11). Healing of 21-dayold skin incisions is impaired in the presence of zinc deficiency, as measured by a significantly decreased wound breaking strength in zinc-deficient rats compared with that of pair-fed controls (12). Zinc, copper and manganese enhance keratinocyte migration in cell cultures using normal human keratinocytes (13), and topical zinc may stimulate leg ulcer healing by enhancing re-epithelialization, decreasing inflammation and bacterial growth, and correcting a local zinc deficit in wound tissue (14). Zinc supplementation may soon be routine in PU patients with low zinc levels.

Albumin concentrations were very significantly lower $(p<0.0001)$ in the PU group. Serum albumin is reported to be lower in tube-fed nursing home patients with pressure sores than in tube-fed patients without sores (15).

Low albumin levels correspond with higher mortality and a more severe disease condition in elderly patients (16). Low albumin levels in animal studies are reported to increase the pressure ulcer risk (17). A multivariate anal- ysis, taking into account all diagnoses and potential risk factors, indicates that low albumin levels at hospital admission represent an independent risk factor for developing a pressure ulcer, and that nutritional intervention lowers the risk (18).

Transferrin levels were significantly $(p<0.0002)$ lower in the PU group. Since transferrin is synthesized in the liver and regulated by the body iron store, these low levels may reflect iron rather than protein deficiency (19).

Plasma iron $(p<0.0001)$ and hemoglobin $(p<0.0002)$ levels were significantly lower in the PU group. Both plasma iron and hemoglobin levels are reported to be lower in tube-fed pressure ulcer patients than in tube-fed patients without pressure ulcers (15). Anemia and iron deficiency in ill elderly patients have been recently reported to be caused partly by malnutrition (20).

Total lymphocyte count was slightly, but not significantly lower in the PU group. The lack of significance may be due to the wide range of normal values or the computational methods used in this analysis. Total lymphocyte count and the CD4/CD8 ratio are decreased in malnourished patients (21).

Plasma cholesterol was significantly lower $(p<0.0001)$ in the PU group. Patients with stage IV pressure ulcers are found to have lower plasma cholesterol levels than patients with stage II or III pressure ulcers (15). Hypocholesterolemia occurs late in the course of malnutrition, which limits the value of cholesterol as an early screening tool. However, it is a major indicator of long-lasting undernutrition (22).

\section{CONCLUSIONS}

The purpose of this study was to compare biochemical nutritional parameters in a group of acutely ill elderly patients who had stage III to IV pressure ulcers with those in a control group of acutely ill patients without pressure ulcers. Both groups had signs of malnutrition, but most nutritional markers were significantly worse 
in the PU group. These findings, while not documenting a causal relationship, suggest a need for routine nutritional assessment and support in acutely ill elderly patients, especially those with pressure ulcers (5).

\section{REFERENCES}

1. Saletti A, Lindgren EY, Johansson L, Cederholm T. Nutritional status according to mini nutritional assessment in an institutionalized elderly population in Sweden. Gerontology 2000; 46: 139-45.

2. Breslow RA, Bergstrom N. Nutritional prediction of pressure ulcers. J Am Diet Assoc 1994; 94: 1301-4.

3. Durkin MT, Mercer KG, McNulty MF, et al. Vascular Surgical Society of Great Britain and Ireland: Contribution of malnutrition to postoperative morbidity in vascular surgical patients. Br J Surg 1999; 86: 702-9.

4. Thomas DR. The role of nutrition in prevention and healing of pressure ulcers. Clin Geriatr Med 1997; 13: 497-511.

5. Thomas DR. Improving outcome of pressure ulcers with nutritional interventions: a review of the evidence. Nutrition $2001 ; 17$ : 121-25.

6. Shea JD. Pressure sores: Classification and management. Clin Orthop Relat Res 1975; 112: 90-100.

7. Smith JD, Burtrimomovitz G, Pudry W. Direct measurement of zinc in plasma by atomic absorption spectroscopy. Clin Chem 1979; 25: 1487-91.

8. Matsuyama N, Takano K, Mashiko T, Jimbo S, Shimetani N, Ohtani $\mathrm{H}$. The possibility of acute inflammatory reaction affects the development of pressure ulcers in bedridden elderly patients. Rinsho Byori 1999; 47: 1039-45.

9. Bonnefoy M, Coulon L, Bienvenu J, Boisson RC, Rys L. Implication of cytokines in the aggravation of malnutrition and hypercatabolism in elderly patients with severe pressure sores. Age Ageing 1995; 24: 37-42.

10. Baez-Franceschi D, Morley JE. Pathophysiology of catabolism in undernourished elderly patients. Z Gerontol Geriatr 1999; 32 (Suppl 1): 12-19.
11. Cruse JM, Lewis RE, Roe DL, et al. Facilitation of immune function, healing of pressure ulcers, and nutritional status in spinal cord injury patients. Exp Mol Pathol 2000; 68: 38-54.

12. Agren MS, Franzen L. Influence of zinc deficiency on breaking strength of 3-week-old skin incisions in the rat. Acta Chir Scand 1990; 156: 667-70.

13. Tenaud I, Sainte-Marie I, Jumbou O, Litoux P, Dreno B. In vitro modulation of keratinocyte wound healing integrins by zinc, copper and manganese. Br J Dermatol 1999; 140: 2634.

14. Agren MS. Studies on zinc in wound healing. Acta Derm Venereol 1990; 154 (Suppl): 51.

15. Breslow RA, Hallfrisch J, Goldberg AP. Malnutrition in tubefed nursing home patients with pressure sores. J Parenter Enteral Nutr 1991; 15: 663-68.

16. Corti M, Guranlik J, Salive M. Plasma albumin level and physical disability as predictors of mortality in older persons. JAMA 1994; 272: 1036-41.

17. Takeda T, Koyama T, Izawa Y, Makita T, Nakamura N. Effects of malnutrition on development of experimental pressure sores. J Dermatol 1992; 19: 602-09.

18. Bourdel-Marchasson I, Barateau M, Rondeau V, et al. A multicenter trial of the effects of oral nutritional supplementation in critically ill older inpatients. GAGE Group (Groupe Aquitain Geriatrique d'Evaluation). Nutrition 2000; 16: 1-5.

19. Payette H, Gray D. Dietary intake and biochemical indices of nutritional status in an elderly population, with estimates of the precision of the 7-d food record. Am J Clin Nutr 1991; 54: 478-88.

20. Mitrache C, Passweg JR, Libura J, et al. Anaemia: an indicator for malnutrition in the elderly. Ann Hematol 2001; 80: 295-98.

21. Chandra RK. Nutrition and immunology: from the clinic to cellular biology and back again. Proc Nutr Soc 1999; 58: 681-83.

22. Omran ML, Morley JE. Assessment of protein energy malnutrition in older persons. Part I: History, examination, body composition, and screening tools. Nutrition 2000; 16: 50-63. 